\title{
Streptococcus dysgalactiae Subspecies Equisimilis; An Agent Rarely Encoun- tered in the Etiology of Septic Arthritis
}

\author{
Meryem Iraz ${ }^{1,{ }^{*}}$, Kerem Bilsel $^{2}$, Hasan Ceylan $^{2}$, Yasemin Akkoyunlu $^{3}$ \\ ${ }_{2}^{1}$ Department of Medical Microbiology, Medical Faculty, Bezmialem Vakif University, Istanbul, Turkey \\ 2 Department of Orthopaedic and Traumatology, Medical Faculty, Bezmialem Vakif University, Istanbul, Turkey \\ 3 Department of Infectious Diseases, Medical Faculty, Bezmialem Vakif University, Istanbul, Turkey \\ ${ }^{*}$ Corresponding author: Meryem Iraz, Department of Medical Microbiology, Medical Faculty, Bezmialem Vakif University, Adnan Menderes Bulvari, Vatan Caddesi, 34093 , Fatih, \\ Istanbul. Tel: +90-2124531700, E-mail: meriraz@mynet.com.
}

Received: November 18, 2012; Revised: January 28, 2013; Accepted: March 03, 2013

\begin{abstract}
Streptococcus dysgalactiae subspecies equisimilis is one of the pyogenic group C and G streptococcus, which may be found in the normal gastrointestinal and genitourinary flora of a healthy human. Many cases have been reported in literature; however, reports of septic arthritis due to this agent without predisposing factors are extremely rare. Diagnosis of this agent is possible in advanced laboratories. Appropriate treatment for septic arthritis caused this agent is made by parenteral antibiotherapy without debridement following accurate diagnosis.
\end{abstract}

Keywords: Streptococcus dysgalactiae; Arthritis, Infectious; Streptococci

\section{Introduction}

Streptococcus dysgalactiae subspecies equisimilis (SDSE) is a large colony-forming human group $C$ and group $G$ streptococci, which belong to the group of pyogenic streptococci frequently referred to as ß-hemolytic Streptococci. SDSE is found in the normal flora of the oropharynx, skin, gastrointestinal and genitourinary systems (1, 2 ). SDSE is known to cause pharyngitis and skin infections, although less commonly, compared to Group A and Group B beta-hemolytic Streptococci. Rarely encountered invasive infections include such as arthritis (1-4). In this report, we present a patient with erythema, swelling and increased heat in the right knee, whose synovial fluid culture revealed growth of SDSE.

\section{Case Presentation}

A 56-year-old patient, admitted to our emergency unit on the third day of her painful right knee. Swelling, erythema and effusion were observed. No history of trauma was reported and there was marked limitation of her joint motion due to the pain. There was also no history of systemic disease nor was there any history of recent infection, and no possible focus of infection was identified in the physical examination conducted. Because of increased dermal heat and erythema of both the knee and anterior crus, cellulitis was thought with at first glance. The patient previously was given some analgesics when no particular abnormality could be identified following physical examination at an external clinical unit. Initial laboratory examination revealed a normal leukocyte count and a slight increase in C-reactive protein (CRP) level. The patient underwent puncture with the intent of surgical decompression due to erythema and swelling of the joint. Gram-positive cocci and abundant polymorphonuclear leukocytes (PMNL) were detected in aspiration fluid. The patient was then referred to our emergency orthopedic clinic for further investigation.

In her laboratory findings, a white blood cell count (WBC) of $9.27 \times 10^{3} / \mu \mathrm{L}$, C-reactive protein (CRP) level of $19.903 \mathrm{mg} / \mathrm{dL}$ and an erythrocyte sedimentation rate (ESR) of $42 \mathrm{~mm} /$ hour. The other biochemistry analyses were found to be within normal ranges. Direct X-ray graphy of the patient's right knee demonstrated osteophytes at the patellofemoral joint (Figure 1). There was also arthrosis of the distal femur and the proximal tibial joint surface. An MRI of the right knee was performed to eliminate any case of chronic disease. A fluid-filled knee joint, thinning of the articular cartilage, and subchondral cysts were observed (Figure 2). A very dense, odorless, deep yellow fluid was extracted from the knee following puncture performed under sterile conditions.

Implication for health policy/practice/research/medical education

Definite identification of the etiologic agent and assessment of antibiotic sensitivity is vital for successful treatment of septic arthritis due to SDSE. The choice of an appropriate antibiotic may contribute in reducing the duration and cost of treatment by decreasing development of resistance, without the need for invasive interventions such as joint debridement.

Copyright (c) 2013, Ahvaz Jundishapur University of Medical Sciences; Licensee Kowsar Ltd. This is an Open Access article distributed under the terms of the Creative Commons Attribution License (http://creativecommons.org/licenses/by/3.0), which permits unrestricted use, distribution, and reproduction in any medium, provided the original work is properly cited. 


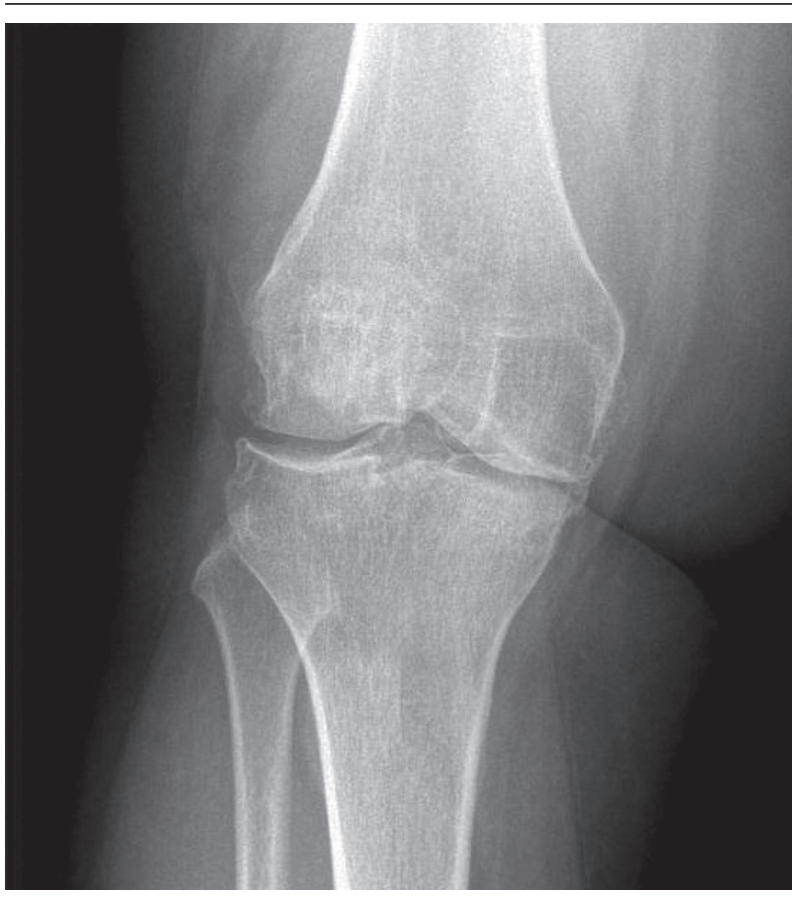

Figure 1. X-ray Examination Demonstrating Marked Osteoarthritis and Periarticular Osteophytes

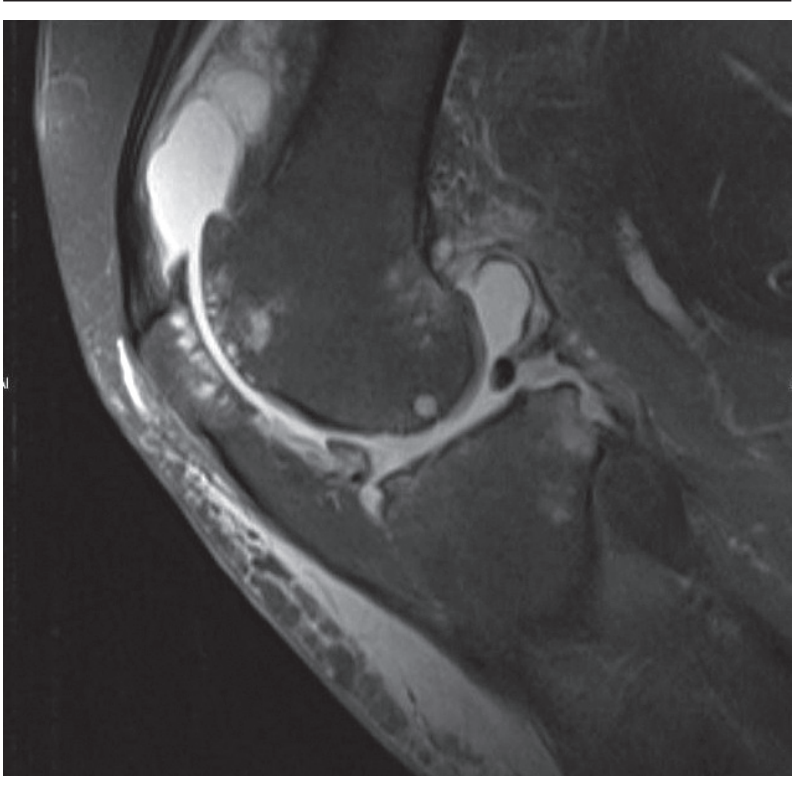

Figure 2. MRI Analysis Demonstrating the Capsule in the Joint Expanded by Marked Joint Effusion

The patient's synovial fluid analysis revealed a leukocyte count of $98.000 / \mathrm{mm}^{3}$ following puncture in the right knee. Direct Gram staining examination on the sample material revealed abundant PMNL and Gram-positive cocci. Simultaneous blood agar and eosin methylene blue agar culture growth of the puncture fluid were performed, and incubated at $35^{\circ} \mathrm{C}$ for $18-24$ hours. The patient was started on empiric ceftriaxone ( 2 g/day) and vancomycin (2 g/day) parenterally. Grayish colonies with large zones of beta-hemolysis were identified on blood agar after incubation. The bacterium isolated from the synovial fluid was identified as catalase-negative gram-positive chains cocci; and evaluated as Streptococcus dysgalactiae subspecies equisimilis by API strep 20 system (Bio Merieux, France) and the automatized systems VITEK2 (Bio Merieux, France). The same bacterium was isolated from the aerobic and anaerobic blood cultures' analysis. Identification and antibiogram results were similar to those of synovial fluid.

Antibiotic sensitivity of the isolated strains was performed according to Clinical and Laboratory Standards Institute criteria, using the Kirby-Bauer disk diffusion test method, with various antibiotic discs (Oxoid, England). The isolate was found to be sensitive to penicillin, ampicillin, clindamycin, cefepime, ceftriaxone, levofloxacin, linezolid, dalfopristin-quinupristin, vancomycin and teicoplanin, whereas it was resistant to chloramphenicol, tetracycline and erythromycin. The antibiotherapy was changed to ampicillin-sulbactam ( $8 \mathrm{~g} /$ day). Three weeks of parenteral antibiotherapy was followed by amoxicillin-clavulanate tablets ( $2 \mathrm{~g} /$ day). Antibiotherapy was then terminated following evaluation of clinical and laboratory recovery by the 6th week of total treatment.

\section{Discussion}

Acute non-gonococcal bacterial arthritis is an infection disease requiring emergency treatment. A full recovery of joint functions is possible when prompt diagnosis is made, and appropriate emergency treatment is provided. Complications such as damage to the joint, osteomyelitis, and periarticular calcification may occur in untreated patients, and death may result particularly in patients with anatomic and functional sequelae (5). Making a diagnosis of reactive synovitis or septic arthritis is not always easy only by physical examination and anamnesis. Laboratory and radiological analysis should also be considered for the diagnosis.

The most common etiologic agent of acute non-gonococcal bacterial arthritis is reported to be Staphylococcus aureus, with $\beta$-hemolytic Streptococci being the second most commonly encountered agents. Group A Streptococcus are said to be the most common bacterial agent involving joints, apart from infection of the joints due to Group B Streptococci reported in diabetic and newborn patients. On the other hand, SDSE infections are very rare, encountered in the community at a rate of 0.003 $0.006 \%$ and among hospitalized patients with a rate of $0.02-0.07 \%$ as the cause of acute non-gonococcal bacterial arthritis $(2,5)$. Most patients with SDSE bacteremia are reported to be the elderly and males, with $74-100 \%$ rates having an underlying disease $(6,7)$.

Although SDSE (Lancefield groups C and G, Streptococci) were recognized as an imported pathogen in hu- 
mans during the early 1980s, very few laboratories could identify them as species. As a result, the exact number of SDSE infections in humans is not well known (1). SDSE primarily causes infections on the skin and soft tissue. Its epidemiology is mostly underestimated, and treatment of its diseases is initiated empirically without bacterial identification. Although these bacteria commonly inhabit the gastrointestinal system, vagina, and the skin of healthy humans, they may cause severe infections in the presence of comorbid diseases such as malignancy, cardiovascular diseases, diabetes mellitus, diseases of the musculoskeletal system, and cirrhosis (3, 4). SDSE may associate with a variety of clinical conditions, including pharyngitis, cellulitis, meningitis, endocarditis, peritonitis, salpingitis, and sepsis. Group A Streptococci-like invasive and systemic infections due to this agent have also been reported $(1,8)$.

The gold standard for the diagnosis of septic arthritis is identification of a microorganism by Gram staining and culture growth of the microorganism, from the purulent synovial fluid. The leukocyte count of the synovial fluid $>$ $50.000 / \mathrm{mm}^{3}$ ( $>80 \% \mathrm{PMNL}$ ) are sufficient to reach a diagnosis, irrespective of the presence or absence of culture growth (9). Analysis of blood culture is also important in the presence of concomitant infectious diseases such as meningitis and endocarditis due to increased mortality rates of about $25 \%$, particularly in elderly patients (1). In this case, a leukocyte count of $98.000 / \mathrm{mm}^{3}(>80 \% \mathrm{PMNL}$ ) was identified in synovial fluid and blood culture growth of SDSE was observed, although there was no predisposing factor and no possible primary focus of infection was detected in contrast to most of the cases in the literature. ESR and CRP are important nonspecific tests practically used in prompt diagnosis and treatment follow-up of bone and joint infections. In a study conducted on patients with septic arthritis, ESR was found to be more than $40 \mathrm{~mm} /$ hour in almost all the patients, while CRP was also found to be positive (10). On the other hand, increase in the joint space and soft tissue swelling as observed through radiologic analysis may contribute to the diagnosis.

In conclusion, definite identification of the etiologic agent and assessment of antibiotic sensitivity is vital for successful treatment of septic arthritis due to SDSE. The choice of an appropriate antibiotic may contribute in reducing the duration and cost of treatment by decreasing development of resistance, without the need for invasive interventions such as joint debridement. Furthermore, our case assumes significance because it occurred during the absence of the characteristic risk factors.

\section{Acknowledgements}

None declared.

\section{Authors' Contribution}

MI initiated the concept, wrote and revised the manuscript. KB and HC involved in writing, creating the illustration and coordination, YA involved in the preparation of manuscript.

\section{Financial Disclosure}

None declared.

\section{Funding/Support}

None declared.

\section{References}

1. Brandt CM, Spellerberg B. Human infections due to Streptococcus dysgalactiae subspecies equisimilis. Clin Infect Dis. 2009;49(5):766-72.

2. Sipahi OR, Ozkoren Calik S, Pullukcu H, Isikgoz Tasbakan M, Arda $\mathrm{B}$, Tunger A, et al. [Streptococcus equisimilis associated septic arthritis/prosthetic joint infection]. Mikrobiyol Bul. 2008;42(3):515-

3. Suemori S, Sawada A, Komori S, Mochizuki K, Ohkusu K, Takemura H. Case of endogenous endophthalmitis caused by Streptococcus equisimilis. Clin Ophthalmol. 2010;4:917-8.

4. Auckenthaler R, Hermans PE, Washington JA, 2nd. Group G streptococcal bacteremia: clinical study and review of the literature. Rev Infect Dis. 1983;5(2):196-204.

5. Gonzalez Teran B, Roiz MP, Ruiz Jimeno T, Rosas J, Calvo-Alen J. Acute bacterial arthritis caused by group C streptococci. Semin Arthritis Rheum. 2001;31(1):43-51.

6. Liu CE, Jang TN, Wang FD, Wang LS, Liu CY. Invasive group G streptococcal infections: a review of 37 cases. Zhonghua Yi Xue Za Zhi (Taipei).1995;56(3):173-8.

7. Skogberg K, Simonen H, Renkonen OV, Valtonen VV. Beta-haemolytic group A, B, C and G streptococcal septicaemia: a clinical study. Scand J Infect Dis. 1988;20(2):119-25.

8. Lopardo HA, Vidal P, Sparo M, Jeric P, Centron D, Facklam RR, et al. Six-month multicenter study on invasive infections due to Streptococcus pyogenes and Streptococcus dysgalactiae subsp. equisimilis in Argentina. J Clin Microbiol. 2005;43(2):802-7.

9. Kabak S, Halici M, Akcakus M, Cetin N, Narin N. Septic arthritis in patients followed-up in neonatal intensive care unit. Pediatr Int. 2002;44(6):652-7.

10. Yorgancigil, H. , Ozerdemoglu, RA. , Bilgen, O. F. , Yorgancigil, B. Septic Arthritis: A review of 64 cases. Hacettepe J Orthop Surgery. 1998;8(1):8-12. 